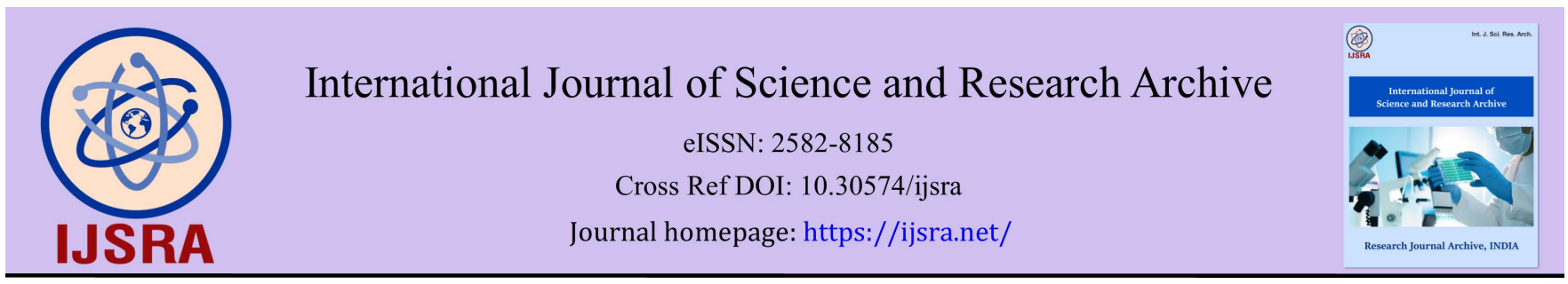

(RESEARCH ARTICLE)

\title{
The effect of 8 weeks swimming training on secretion of growth hormone in 9-11 years old boys
}

\author{
Sepehri Manesh Mohammad 1,2, ${ }^{*}$, Motaghi Hamze ${ }^{2}$ and Abasi Moghadam Mahdi ${ }^{1}$ \\ ${ }^{1}$ Khorramabad Technical and Vocational College, Khorramabad, Lorestan, Iran. \\ ${ }^{2}$ Lorestan organization of Education, Khorramabad, Lorestan, Iran.
}

International Journal of Science and Research Archive, 2021, 02(02), 139-144

Publication history: Received on 13 April 2021; revised on 16 May 2021; accepted on 20 May 2021

Article DOI: https://doi.org/10.30574/ijsra.2021.2.2.0069

\begin{abstract}
The influence of exercise training on anabolic hormones is especially important for growth and development in childhood years. The aim of this study was determine effect of 8 weeks of swimming training on secretion of growth hormone in 9-11 years old boys. To test the research hypothesis, the people who were willing to cooperate with the investigator randomly assigned to experimental group ( $n=15$ : age: $9.33 \pm 0.30$, height: $130.46 \pm 7.53$, weight: $27.04 \pm 7.39$ ) and control group ( $\mathrm{n}=15$ : age: $9.39 \pm 0.39$, height: $133.53 \pm 7.52$, weight: $27.15 \pm 4.92$ ). Experimental group participated in 8 weeks swimming training with intensity of $45-65 \%$ HRmax for 3 times per week and 1 hour per session. During this time control group did not participate in this protocol and continue to routine activity. Fasting blood sample were collected 48 hours before and after of 8 weeks swimming training to assess serum growth hormone. 8 weeks swimming training led to significant increase on growth hormone in experimental group $(\mathrm{P}=0.000)$. But increase growth hormone in control group not significant $(\mathrm{P}=0.383)$. In result, 8 week of swimming training led to significant increase on secretion growth hormone in 9-11 years old boys.
\end{abstract}

Keywords: Somatotropin; Swimming training; Physical activity

\section{Introduction}

For all people dealing with children, it is important to be familiar with the growth process and the effects of various factors on it. On the other hand, physical activity is very effective in the lifestyle of children and adolescents and can be manifested in various forms including free play and sports, physical education in school and organized sports. Many researchers also believe that regular physical activity during adolescence has beneficial and long-term effects on a person's health in adulthood [1]. Unfortunately, the negative consequences of inactivity have spread in today's society. It seems that today, more than ever, there is a need to improve children's lifestyle patterns. In the meantime, it is vital to know all the factors that affect the health, growth and vitality of the community. Growth Hormone (GH) is one of the factors affecting the growth of children. This hormone stimulates the growth of all tissues in the body [2]. Growth hormone affects the bone growth plate, and the high secretion of this hormone before the growth plate closes causes a giant and after the growth plate closes, it causes acromegaly, which means that the organs of the nose and chin widen and the forehead protrudes. Decreased release of this hormone causes dwarfism [3]. Of course, the main effect of growth hormone is on protein synthesis. Thus, growth hormone in childhood stimulates the growth of bones and muscle tissue, and by hypertrophy and hyperplasia in various types of cells, including skeletal muscle myoblasts and tendon fibroblasts, the size and characteristics of a person's body Determines [4-5].

\footnotetext{
${ }^{*}$ Corresponding author: Mohammad Sepehri Manesh

Khorramabad Technical and Vocational College, Khorramabad, Iran. 
Considering the effects of growth hormone on the growth process of children, researchers have paid attention to what factors can affect the release of growth hormone, and many studies have been done to identify these factors. Normal growth in children is largely influenced by the action of growth hormone and insulin-like growth factor (IGF-1). Two very influential factors on this axis are nutrition and physical activity. Various studies show that exercise can affect the release of growth hormone so that both aerobic exercise and resistance exercise can lead to increased growth hormone release [6]. Of course, hormonal responses to exercise vary at different ages. Children before puberty do not show the same and predictable responses to exercise due to the lack of development of some physiological systems. Exercise has many benefits and the necessary measures to increase physical activity throughout life should be taken [7]. Researchers in various studies have concluded that physical activity during childhood can increase the risk of obesity [8-10], osteoporosis [11-12], cardiovascular disease [13], diabetes [14] Reduce high blood pressure [15] and metabolic syndrome [16] in adulthood. Regular exercise also increases aerobic fitness in children and adults [16]. Despite these results, the harmful effects of intense exercise on growth, skeletal maturity and pubertal development in various sports have been studied [17]. Studies in children have shown that exercise can stimulate both growth factor (such as growth hormone) and stress factor (such as cytokines), which have opposite functions [18]. Although most studies have reported the positive effect of exercise on growth, some studies report conflicting results [19-20]. Therefore, understanding the factors affecting growth requires more research, so that we can prescribe appropriate activities for children and adolescents, taking into account all possible aspects. Of course, the type of exercise selected also affects the growth hormone response to exercise. Most previous research has examined acute growth hormone responses to exercise, but results on long-term responses to exercise in children and adolescents are very limited and require further research.

Evidence suggests that exercise that engages the large muscles further enhances the release of growth hormone [21]. On the other hand, the purpose of choosing swimming exercise in this study was that this exercise, in addition to creating freshness and vitality among children, involves the large muscles of the body. In the present study, the effect of 8 weeks of swimming training on the secretion of growth hormone in 9 to 11-year-old boys was investigated.

\section{Material and methods}

The subjects of this study are 9 to 11-year-old boys in Khorramabad city who were familiar with crawl swimming (performing the correct crawl technique) and were willing to cooperate with the researcher. 87 of them expressed their consent to participate in the research after completing the consent form. These individuals were then given a medical exercise history questionnaire and asked to complete it. Inclusion criteria included: being 9 to 11 years old and having physical health and basic familiarity with crawl swimming, and not attending regular and club exercises and no history of acute and chronic metabolic, cardiovascular and genetic diseases. After reviewing the questionnaires and initial examinations by a general practitioner, 4 subjects were excluded from the study at the physician's discretion. The reason for excluding these 4 subjects was that 2 of them had cardiovascular disorders and the other two subjects were diagnosed Physicians did not grow normally to their age. Then, 30 were selected for the study. Among them, 15 were selected for the control group and 15 for the exercise group by random selection. 48 hours before the start of training on an empty stomach and 48 hours before the exercise, intense physical activity was not performed by the subjects, blood samples were taken by a laboratory specialist from both control and practice groups. 48 hours after sampling the training group, they started the swimming training program. Finally, 48 hours after 8 weeks of swimming training performed by the training group, for the second time from both research groups, blood samples were taken by a laboratory specialist similar to the pre-test situation.

In addition to their daily activities, the training group performed 8 weeks of swimming exercises, which lasted 3 sessions per week and an average of 60 minutes per session. Swimming exercise included 10 minutes of warm-up, 45 minutes of chest crawl swimming with an intensity of 45 to $65 \%$ of maximum heart rate, and five minutes of cooling. Warming up included stretching and flexing out of the water and walking and dedicated warm-up in the water. Cooling also included playing in the water. The method of controlling the intensity of training was as follows:

Formula: maximum heart rate $=$ age -220

The maximum heart rate of the subjects was calculated and its range was 45 to $65 \%$, then at rest it was controlled using a polar heart rate monitor. Subjects walked across the pool and then rested. By measuring the subjects' heart rate, the rhythm of the activity was determined and the rest between the activities was determined. Subjects (both practice group and control group) were asked to continue their daily activities outside the research hours as in the past.

In the present study, the following tools and instruments were used for the required measurements: 1 - Medical Sports History Questionnaire 2- Scales 3- Tape Meters 4- Growth Hormone Kits 5- Polar Pulse Meter. The questionnaire used 
in this research was researcher-made and was designed according to the nature of the research and the information required for it. A Burer digital scale with a sensitivity of $\geq 10 \mathrm{~g}$ was used to measure the weight of the subjects. A tape measure was used to measure the height of the subjects. A tape measure was mounted on the wall and a height was measured by placing a ruler on the subjects' heads. To determine the amount of growth hormone in each blood sample, use the Growth Hormone ELISA kit made by Biosensis with a sensitivity of $<2 \mathrm{pg} / \mathrm{ml}$ and enzyme-dependent immunological uptake assay or ELISA was used. All steps of growth hormone measurement were performed in the laboratory. To measure the heart rate of the subjects, the Polar F4 heart rate monitor made in Finland was used.

Descriptive statistics were used to describe the subjects. The data are shown as standard deviation \pm mean, ANOVA test with repeated measures and paired t-test and independent t-test were used for statistical analysis of data. SPSS 19 software was used to perform statistical methods and a significance level of $\mathrm{P}<0.05$ was considered.

\section{Results}

The descriptive characteristics of the subjects including height, body mass and Body Mass Index (BMI) of the control and experimental groups as the mean and standard deviation ( $\mathrm{m} \pm \mathrm{SD}$ ) are shown in Table (1).

Table 1 Description of height, mass and body mass index of the subjects

\begin{tabular}{l|l|l|}
\hline \multicolumn{1}{l}{ Variable } & \multicolumn{1}{l}{ Control group } & Experimental group \\
\hline Height $(\mathrm{cm})$ & $133.53 \pm 7.52$ & $130.46 \pm 7.53$ \\
\hline Weight $(\mathrm{kg})$ & $27.15 \pm 4.92$ & $27.04 \pm 7.39$ \\
\hline Age(year) & $9.39 \pm 0.39$ & $9.33 \pm 0.30$ \\
\hline $\mathrm{kg} / \mathrm{m}^{2}(\mathrm{BMI})$ & $1.38 \pm 15.17$ & $1.38 \pm 15.17$ \\
\hline
\end{tabular}

In addition, ANOVA test with repeated measures showed a significant effect of time $(\mathrm{P}=0.000 ; 287=57.7$ and 1$) \mathrm{F}$ significant effect of time and group $(\mathrm{P}=0.000 ; 28=1560) \mathrm{F}$ and the effect of the group was significant $(\mathrm{P}=0.000 ; 273.9$ $=28$ and 1$)$, Was $(\mathrm{P}=0.000 ; 14=24.3)$ but the increase of $0.5 \%$ in the control group was not significant $(\mathrm{P}=0.384 ; \mathrm{t}=$ $-0.9) \mathrm{T}$-test showed no significant difference in growth hormone levels between the groups in the pre-test $(\mathrm{P}=0.474 ; \mathrm{t}$ $=-0.726)$ and a significant difference in the post-test $(\mathrm{P}=0.000 ; 298) .21=28)$.

Table 2 Mean and standard deviation of growth hormone values in pre-test and post-test in $\mathrm{ng} / \mathrm{ml}$

\begin{tabular}{|l|l|l|l|}
\hline \multicolumn{2}{|l|}{ Control group } & \multicolumn{2}{l|}{ Experimental group } \\
\hline Pre test & Post test & Pre test & Post test \\
\hline $17.47 \pm 0.3$ & $17.51 \pm 0.4$ & $17.38 \pm 0.3$ & ${ }^{*} 23.13 \pm 0.9$ \\
\hline
\end{tabular} *. Significant difference between pre-test and post-test values of control group $(\mathrm{P}=0.000)$

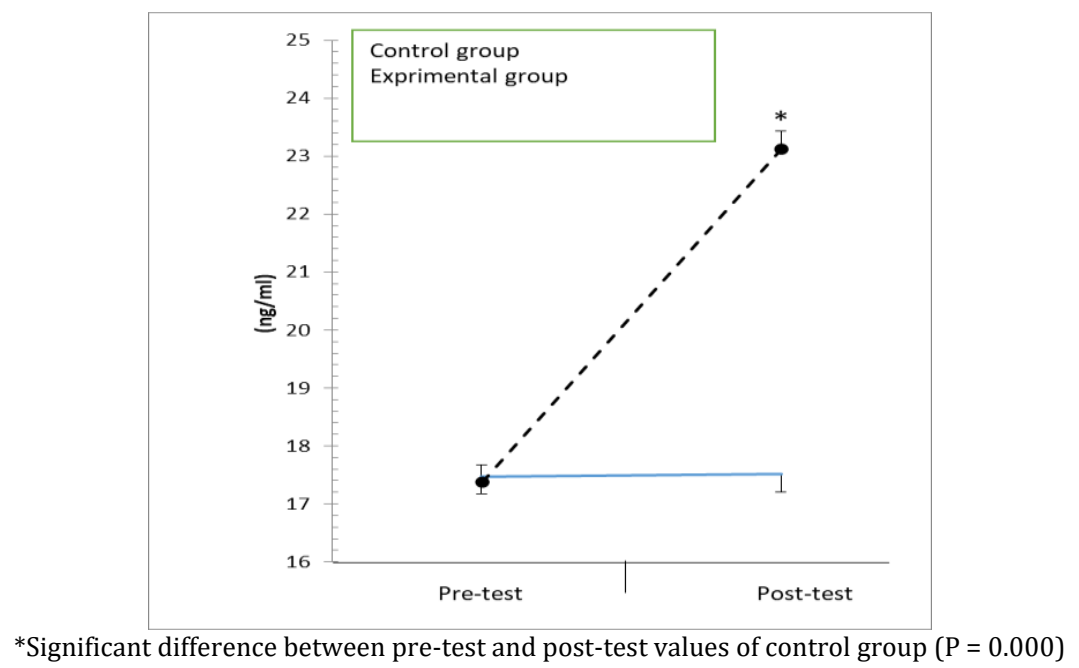

Figure 1 Change in growth hormone levels in experimental and control groups from pre-test to post-test 


\section{Discussion}

The aim of this study was to investigate the effect of 8 weeks of swimming training on the secretion of growth hormone in boys aged 9 to 11 years. The results showed that after 8 weeks, growth hormone was increased in both groups, but this increase was significant only in the exercise group. Therefore, these results indicate that 8 weeks of swimming training significantly increases growth hormone in 9 to 11-year-old boys.

In previous research, various cases have been suggested for possible mechanisms of increase in growth hormone after exercise, which vary depending on the type of exercise selected and the factors affecting it. We now turn to some of these possible mechanisms. Anabolic and catabolic phase, the duration of exercise is one of the factors affecting the response of growth hormone to exercise, so that it has been observed that if the duration of exercise is less than 5 weeks leads to catabolic response and if it is more than 5 weeks leads to anabolic response [22]. Now, considering the 8-week period of this study, it seems that the anabolic effect of exercise has probably been further stimulated. The type of exercise, due to the choice of swimming discipline for this study and the involvement of large muscles of the body, the response of growth hormone to exercise has been more. The positive effect of large muscle involvement in exercise on growth hormone has been confirmed in other studies [23]. Another mechanism is a change in stimuli that release growth hormone [20]. GHRH is one of the factors that can be influenced by other hormonal factors such as catecholamines [20]. Of course, because only growth hormone has been studied in this study, it can not be named as a definitive mechanism in this study and further research is needed in the future. Also, it has been found that healthier people have higher levels of growth hormone [24]. Although health indicators have not been studied in this study, but the effect of exercise on health and well-being of individuals has been fully confirmed [25-26-27-28] and can be considered as one of the factors increasing growth hormone in the present study. The cause of acute and chronic effects of exercise, previous studies have shown, growth hormone increases in response to different exercises, but the type of response is different [29]. Single-session studies and studies that examined growth hormone release immediately after exercise have shown that such exercise leads to an increase in growth hormone responsive responses, while long-term exercise, stimulate growth hormone biological and anabolic responses for several weeks by adapting to the individual [29].

Consistent with the results of the present study, Widman et al. (2006) stated that vigorous stimulation exercise is for the release of growth hormone and both aerobic exercise and resistance exercise lead to a sharp increase in growth hormone release [30,31]. A very important point to note is that most research has dealt with the acute effect of growth hormone, and this alignment of the results should not lead to misguidance in the absolute emphasis of the present research results. Buyukyazi, et al. (2003) reported in a study that eight weeks of aerobic exercise had an effect on adolescent boys' growth hormone [31]. The results of this research are in line with the present research while the type of research is largely similar to the present research. According to the training protocol of this study and the present study, which stimulate both large muscles of the body, one of the reasons for the similarity of the results can be related to the muscles involved. In addition, Bosco, et al, as well as Smilios, et al., Reported that strength training performed to maximize strength would not significantly increase post-workout growth hormone release [31]. One reason for the discrepancy between the results is that these studies have different training and subject protocols than the present study. Another reason for this discrepancy may be attributed to the training history. Given that the exercise was performed in order to gain maximum strength, the participants in the study certainly had a previous history of exercise, and as mentioned earlier, experienced people show less response to exercise.

\section{Conclusion}

Overall, the results of this study showed that 8 weeks of swimming training can stimulate the release of growth hormone in 9 to 11-year-old boys. Despite extensive research on the effects of exercise on the endocrine glands, including the present study, valuable information has been obtained on the extent of changes in hormones, including growth hormone, but the findings are contradictory. It needs further examinations inevitable, especially in children and adolescents.

\section{Compliance with ethical standards}

\section{Acknowledgments}

The authors wish to acknowledge with gratitude the authors whose works were extensively used in this study and duly acknowledge in the reference section. 


\section{Disclosure of conflict of interest}

The authors declare no conflict of interest in the present study.

\section{Statement of informed consent}

Informed consent was obtained from all individual participants included in the study.

\section{References}

[1] Roberts Robert A, Roberts Scott A. Basic principles of sports physiology. Publisher: Mosby College (September 1, 1999); Language: English; Paperback: 544.

[2] Guyton, Arthur, Hall John Edward. Guyton Medical Physiology (Eleventh Edition), Guyton and Hall Textbook of Medical Physiology, 13th Edition. Reviewed in the United States on April 2, 2018.

[3] Vishram Singh. General anatomy. Third Edition, eBook ISBN: 9788131262443.

[4] Gaini, Abbas Ali. Human physiology. second edition. ISBN: 978-1-119-48486-8, January 2019, 944 Pages.

[5] Abrahamsson SO. Similar effects of recombinant human insulin-like growth factor-I and II on cellular activities in flexortendons of young rabbits: experimental studies in vitro. J Orthop Res. 1977; 15: 256-262.

[6] Wideman L, Consitt LA, Patric J. the impact of sex and exercise duration on growth hormone secretion .J appl physiol. 2006; 1641-1647.

[7] Silverstein J, Klingensmith G, Copeland K, Plotnick L, Kaufman F, Laffel L, et al. Care of Children and Adolescents With Type 1 Diabetes. Diabetes Care. 2005; 28(1): 186-212.

[8] Steinberger J, Daniels SR, Eckel rh, Hayman l, Lustig RH, McCrindle B, et al. Progress and Challenges in Metabolic Syndrome in Children and Adolescents. Circulation. 2009; 119: 628- 647.

[9] Jurimae T, Jurimae J, Maestu J. Metabolic syndrome, physical activity and adipocytokines in children and adolescents. Med Sport. 2009; 13(1): 22-27.

[10] Daniels SR, Arnett DK, Eckel RH, Gidding SS, Hayman LL, Kumanyika S, et al. Overweight in Children and Adolescents: Pathophysiology, Consequences, Prevention, and Treatment. Circulation. 2005; 111: $1999-2012$.

[11] Dickson C. What is a girl`s experience of physical activity? A qualitative descriptive study [Dissertation]. Auckland Univ. 2008.

[12] Outila TA, Karkkainen MUM, Lamberg- Allardt CJE. Vitamin D status affects serum parathyroid hormone concentrations during winter in female adolescents: associations with forearm bone mineral density. Am J Clin Nutr. 2001; 74: 206-210.

[13] Carrel AL, McVean JJ, Clark RR, Peterson SE, Eickhoff JC, Allen DB. School-based Exercise Improves Fitness, Body Composition, Insulin Sensitivity, and Markers of Inflammation in Non-Obese Children.J Pediatr Endocrinol Metab. 2009; 22: 409-415.

[14] Carson VL. Screen time and seasonal variation in physical activity among preschool children in Edmonton [Dissertation]. Alberta Univ. 2009; 169.

[15] Dong-IL seo, Tae-won Jun, Kae-Soon Park, Hyukki Chang, Wi-Young So, Wook Song. 12 Weeks of Combined Exercise Is Better Than Aerobic Exercise for Increasing Growth Hormone in Middle-Aged Women. International Journal of Sport Nutrition and Exercise Metabolism. 2010; 20: 21-26.

[16] Jurimae T, Jurimae J, Maestu J. Metabolic syndrome, physical activity and adipocytokines in children and adolescents. Med Sport. 2009; 13(1): 22-27.

[17] Georgopoulos NA, Markou KB, Theodoropoulou A, Benardot D, Leglise M, Vagenakis AG. Growth retardation in artistic compared with rhythmic elite female gymnasts. J Clin Endocrinol Metab. 2002; 87: 3169-3173.

[18] Zaldivar F, Wang-Rodriguez J, Nemet D, Schwindt C, Galassetti P, Mills PJ, et al. Constitutive pro- and antiinflammatory cytokine and growth factor response to exercise in leukocytes. J Appl Physiol. 2006; 100: 1124 1133.

[19] Eliakim AP, Scheett T, Newcomb R, Mohan S, Cooper DM. Fitness training and the growth hormone-Insulin-like growth factor I axis in prepubertal girls. J. Clin. Endocrinol. Metab. 2001; 86: 2797-2802. 
[20] Kate L Gilbert, Keith A Stokes, George M Hall, Dylan Thompson. Growth hormone responses to 3 different exercise bouts in 18- to 25- and 40- to 50-year-old men. Appl Physiol Nuter Metab. 2008; 33: 706-712.

[21] Kraemer William J, Bradley C Nindl, James O Marx, Lincoln A Gotshalk, Jill A Bush, Jill R Welsch, Jeff S Volek, Barry A Spiering, Carl M Maresh, Andrea M Mastro, Wesley C Hymer. Chronic resistance training in women potentiates growth hormone in vivo bioactivity: characterization of molecular mass variants. Human Performance Laboratory, Dept. of Kinesiology, Univ. of Connecticut, Storrs, CT 06269. 2006.

[22] Cristiane da Silva C, Goldberg TBL, dos Santos Teixeira A, Marques I. Does physical exercise increase or compromise children's and adolescent's linear growth? Is it a myth or truth? Rev Bras Med Esport. 2004; 10(6): 525- 528.

[23] Iedda Brasil, et al. Effects of judo training upon body composition, autonomic function, and cardiorespiratory fitness in overweight or obese children aged 8- to 13 years. Journal of Sports Sciences. 2020; 38(21).

[24] Zhigoang Zhang, Nicholas Kuzik. Associations between the Child Care Environment and Children's In-Care Physical Activity and Sedentary Time.health education journal. 2021; 48(1): 42-53.

[25] Daniels SR, Arnett DK, Eckel RH, Gidding SS, Hayman LL, Kumanyika S, et al. Overweight in Children and Adolescents: Pathophysiology, Consequences, Prevention, and Treatment. Circulation. 2005; 111: 1999-2012.

[26] Dickson C. What is a girl`s experience of physical activity? A qualitative descriptive study [Dissertation]. Auckland Univ. 2008.

[27] Wideman L, Consitt LA, Patric J. The impact of sex and exercise duration on growth hormone secretion .J appl physiol. 2006; 1641-1647.

[28] Poehlman ET, Copeland KC. Influence of physical activity on insulin-like growth factor-I in healthy younger and older men. J Clin Endocrinol Metab. 1990; 71: 1468-1473.

[29] Buyukyazi G, Karamizrak S, Islegen C. Effects of continuous and interval running training on serum growth and cortisol hormones in junior male basketball players. Acta physiol Hing. 2003; 90(1): 69-79.

[30] Bosco G Colli, Bonmi R. Monitoring strength training neuromuscular and hormonal profile. Med sci sport exercise. 2000; 32: 202-8.

[31] Smilios I, pilianidis Y, karamouzis M, parlavantzas A, Tokmakidis SP. Hormonal response after a strength endurance resistance exercise protocol in young and elderly males. Inta sports med. 2006; 763-784. 